\title{
Simultaneous Reciprocal and Real Space X-Ray Imaging of Time-Evolving Systems
}

\author{
Matias Kagias $\odot,{ }^{1, *}, \dagger$ Zhentian Wang $\odot,{ }^{1,2}$ Goran Lovric $\odot,{ }^{1,3}$ Konstantins Jefimovs, ${ }^{1}$ and \\ Marco Stampanoni ${ }^{1,2}$ \\ ${ }^{1}$ Swiss Light Source, Paul Scherrer Institute, Villigen 5232, Switzerland \\ ${ }^{2}$ Institute for Biomedical Engineering, University and ETH Zurich, Zurich 8092, Switzerland \\ ${ }^{3}$ Centre d'Imagerie BioMédicale, École Polytechnique Fédérale de Lausanne, Lausanne 1015, Switzerland
}

(Received 23 November 2020; revised 12 February 2021; accepted 7 April 2021; published 23 April 2021)

\begin{abstract}
Imaging the (sub)micron scale over large areas with high temporal resolution becomes increasingly necessary for the development and investigation of novel materials under realistic operation conditions. Small angle x-ray scattering imaging methods provide micro- and nanoscale structural information of materials. A fundamental shortcoming of such methods is the long acquisition time required to investigate macroscopic objects. In this work, we propose a single shot imaging method that allows reciprocal space sensitivity at a local level while maintaining spatial resolution for imaging macroscopic objects. We use an instrument that is sensitive to the ultrasmall angle $\mathrm{x}$-ray scattering range and utilize it to image unstable polydisperse particle systems. This allows us to observe in real time the evolution of the local average particle diameter due to the stratification of the microparticles.
\end{abstract}

DOI: 10.1103/PhysRevApplied.15.044038

\section{INTRODUCTION}

Understanding and manipulating the structure of soft and condensed matter at (sub)micrometer scales has led to the development of novel materials with highly optimized properties. Eminent examples include ultralight metallic microlattices [1], catalyst supports [2], and battery electrodes [3]. The development and improvement of such materials requires appropriate methods for sensing or visualizing the (sub)micron scale. For example, in the field of colloids and microparticle suspensions state-of-the-art methods include $\mathrm{x}$-ray diffraction and scattering studies $[4,5]$ or microscopic techniques resolving directly individual particles [6,7]. Both approaches cannot fulfill simultaneously the requirements for realistic operando investigations, i.e., high temporal resolution, large imaging area, and (sub)micrometer-scale sensitivity.

Modulator-based x-ray imaging methods, such as grating interferometry [8], coded apertures [9], and specklebased [10] imaging, can provide subpixel information over large areas by retrieving scattering-based images [11-15]. Furthermore, the utilization of $\mathrm{x}$ rays allows one to investigate thick, turbid, and opaque samples, and also enables access to three-dimensional information. A major

\footnotetext{
*mkagias@caltech.edu

$\dagger$ Currently at Division of Engineering and Applied Science, California Institute of Technology, Pasadena, CA 91125, USA.
}

shortcoming of the current approaches is that, for quantification of the microstructure (i.e., characteristic length, orientation, etc.), multiple measurements are needed [16-20]. Hence, in the case of time-evolving, spatially modulated systems the state-of-the-art methodology is limited.

In this work, we propose a method that can deliver quantitative subpixel information of macroscopic objects in a single shot. The method utilizes a diffractive x-ray optical element that is subdivided into identical unit cells, with each unit cell designed such that it provides locally reciprocal space information [sensing the (sub)micrometer scale] and acts as a macropixel in the final image, thus maintaining spatial resolution. This approach can be operated in a single shot mode, enabling a high temporal resolution. Additionally, the scalability of the method is straightforward since the sample size is only limited by the available x-ray beam. As unit cells, we choose $\pi$ phase shifting Fresnel zone plates that act as a far-field transformation [21].

\section{SIMULTANEOUS REAL AND RECIPROCAL SPACE IMAGING}

In Fig. 1(a) an implementation of the proposed method is shown. The sample is placed right before the optical element that is composed of an array of zone plates with radius $R_{N}$, spacing $\Delta X$, focal length $f$, and most outer zone width $\Delta r$. An x-ray detector with a physical pixel size 


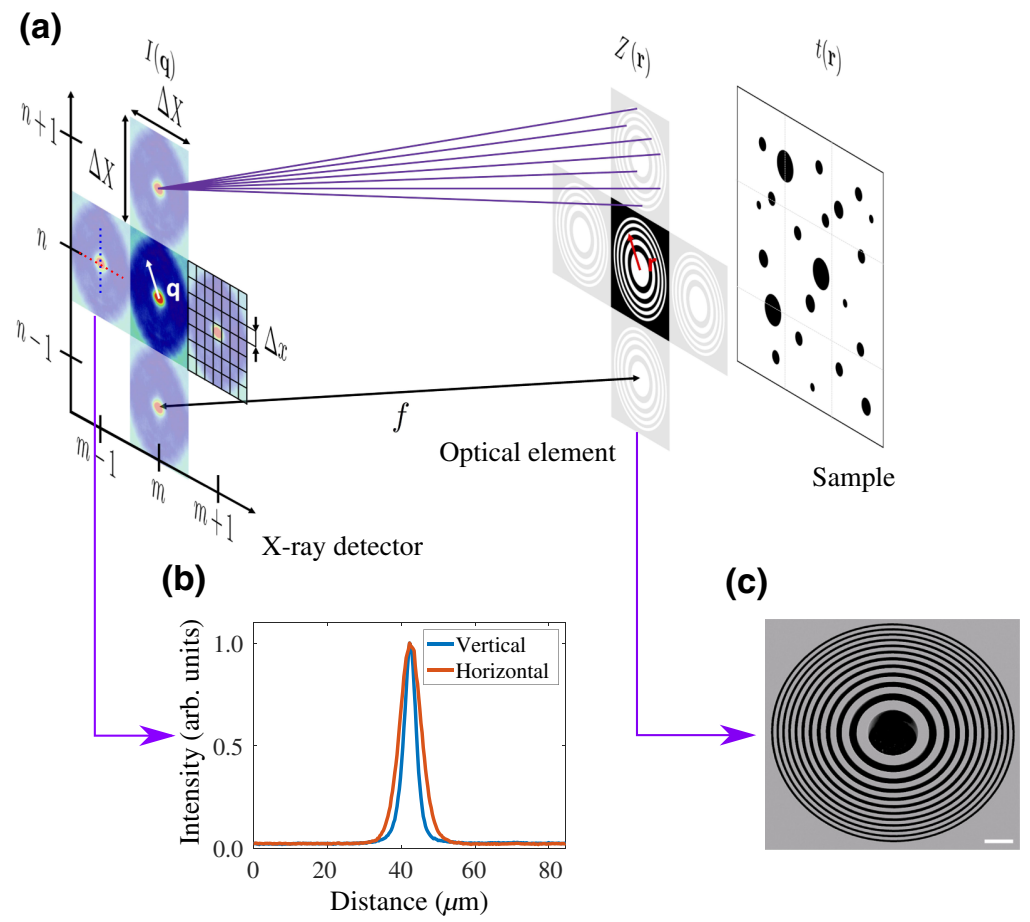

FIG. 1. Experimental setup for simultaneous real and reciprocal space imaging. (a) The sample $t(\mathbf{r})$ is placed right before the Fresnel zone plate $Z(\mathbf{r})$. The x-ray detector is placed at the focal plane of the zone plates. The focusing of the zone plates is equivalent to recording the far-field image $I(\mathbf{q})$ of the probed area of the sample, meaning that reciprocal space information is recorded in each unit cell $(m, n)$. (b) Horizontal and vertical line profiles at the central part of the recorded intensity pattern. (c) Scanning electron microscopic image of a single unit cell. The outer zone width is $1.2 \mu \mathrm{m}$ and the diameter is $84.5 \mu \mathrm{m}$. The scale bar is $10 \mu \mathrm{m}$.

$\Delta x$ is placed at the focal plane of the Fresnel zone plate array. The number of physical detector pixels per unit cell is $N$; without loss of generality, we assume that $N$ is odd. In the Appendix we establish the connection between the physical parameters of the zone plates and the scattering range that is sensed. The norms of the maximum and minimum scattering vectors are given by $q_{\max }=2 \pi(1 / \Delta r)$ and $q_{\min }=2 \pi(1 / \Delta r)(2 / N-1)$ respectively. This range can also be interpreted in real space by the correlation lengths $\xi$; the maximum and minimum are $\xi_{\max }=(N-1 / 2) \Delta r$ and $\xi_{\min }=\Delta r$, respectively. The diameter of the zone plates is limited by their spacing $2 R_{N} \leq \Delta X$ and therefore we end up with a fundamental trade-off between real space resolution $\Delta X$ and the resolution of characteristic length scales:

$$
\Delta X \Delta \xi \geq \lambda f
$$

The recorded scattering patterns are convolved by the source distribution and the point spread function of the detector; this hinders an analysis directly in the reciprocal space. To overcome this, we retrieve the real space correlation function $[22,23]$ instead as conventionally done in grating-based imaging $[16,24]$. For each unit cell $(m, n)$, we record a sample and flat diffraction pattern, denoted by $I_{s}^{(m, n)}\left[q_{k}^{x}, q_{l}^{y}\right]$ and $I_{f}^{(m, n)}\left[q_{k}^{x}, q_{l}^{y}\right]$, respectively, where $q_{k}^{x}$ and $q_{l}^{y}$ are the discrete coordinates with $i, k=-(N-$ $1) / 2, \ldots,(N-1) / 2$. The flat measurement is performed in order to correct for the blurring effects. The local sample transmission can be retrieved from the zeroth components of the discrete Fourier transforms for each recorded pattern:

$$
T[m, n]=\frac{\hat{I}_{s}^{(m, n)}\left[\xi_{0}^{x}, \xi_{0}^{y}\right]}{\hat{I}_{f}^{(m, n)}\left[\xi_{0}^{x}, \xi_{0}^{y}\right]} .
$$

TABLE I. Experimental parameters for the two demonstrated experiments.

\begin{tabular}{lcccccccc}
\hline \hline & $\begin{array}{c}\Delta r, \Delta \xi, \xi_{\min } \\
(\mu \mathrm{m})\end{array}$ & $\begin{array}{c}R_{N} \\
(\mu \mathrm{m})\end{array}$ & $\begin{array}{c}\Delta X \\
(\mu \mathrm{m})\end{array}$ & $\begin{array}{c}\Delta x \\
(\mu \mathrm{m})\end{array}$ & $\begin{array}{c}E(\mathrm{keV}) \\
(\mathrm{keV})\end{array}$ & $\begin{array}{c}\xi_{\max } \\
(\mu \mathrm{m})\end{array}$ & $\begin{array}{c}f \\
(\mathrm{~m})\end{array}$ & $\begin{array}{c}t \\
(\mathrm{~ms})\end{array}$ \\
\hline Validation experiment & 1.2 & 42.25 & 84.5 & 6.5 & 17 & 7.2 & 1.3 & 10 \\
Dynamic experiment & 0.82 & 42.25 & 84.5 & 6.5 & 25 & 4.9 & 1.3 & 10 \\
\hline \hline
\end{tabular}



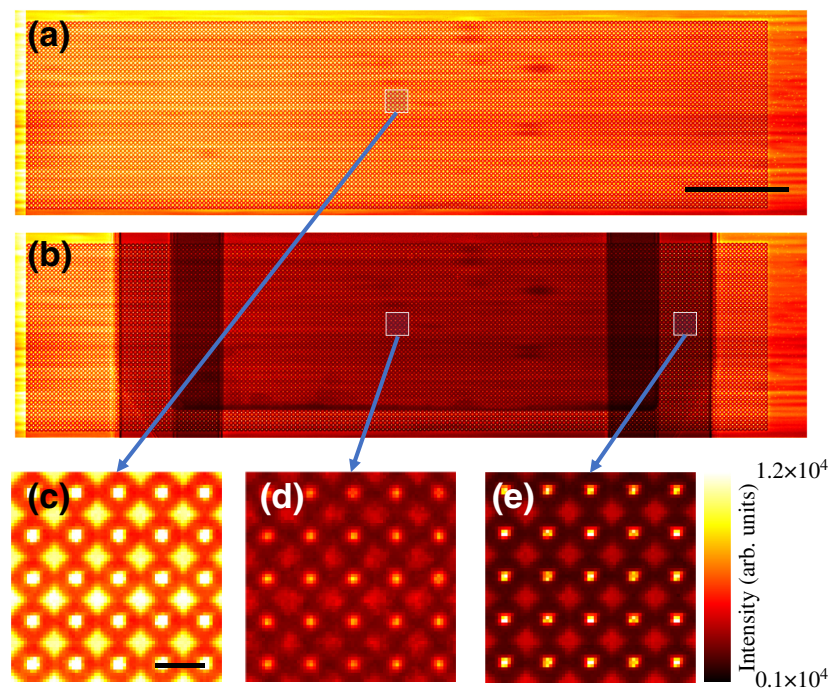

FIG. 2. Raw images for the validation experiment. (a) Flat and sample (b) images for the $7.7 \mu \mathrm{m}$ particles (scale bar $2.5 \mathrm{~mm}$ ). (c),(d),(e) Enlargements of the marked areas. The scale bar is $170 \mu \mathrm{m}$.

The real space correlation function $\Gamma^{(m, n)}\left[\xi_{k}^{x}, \xi_{l}^{y}\right]$ is given by

$$
\Gamma^{(m, n)}\left[\xi_{k}^{x}, \xi_{l}^{y}\right]=\frac{\hat{I}_{s}^{(m, n)}\left[\xi_{0}^{x}, \xi_{0}^{y}\right]}{\hat{I}_{f}^{(m, n)}\left[\xi_{0}^{x}, \xi_{0}^{y}\right]} \frac{\hat{I}_{s}^{(m, n)}\left[\xi_{k}^{x}, \xi_{l}^{y}\right]}{\hat{I}_{f}^{(m, n)}\left[\xi_{k}^{x}, \xi_{l}^{y}\right]} .
$$

Since we image isotropically scattering structures, we report on the radially integrated real space correlation function

$$
\Gamma^{(m, n)}\left[\xi_{k}^{\rho}\right]=\frac{2}{N-1} \sum_{l=1}^{(N-1) / 2} \Gamma^{(m, n)}\left[\xi_{k}^{\rho}, \theta_{l}\right],
$$

where $\xi_{k}^{\rho}$ and $\theta_{l}$ are discrete polar coordinates.

\section{EXPERIMENTAL VALIDATION}

To demonstrate the working principle of the proposed method, we fabricated a zone plate array with a total number of 8418 individual zone plates arranged in a $183 \times$ $46(\mathrm{~h} \times \mathrm{v})$ grid covering a total area of $1.57 \times 0.38 \mathrm{~cm}^{2}$. The design parameters of this validation experiment are summarized in Table I. A scanning electron micrograph of one of the zone plates is shown in Fig. 1(c). For the fabrication, we utilized electron beam lithography and deep reactive ion etching of $\mathrm{Si}$. The details of the fabrication and characterization are given in the Supplemental Material [25]. The utilized detector is the pco.edge 5.5 camera with a pixel size of $6.5 \mu \mathrm{m}$. The $\mathrm{x}$ rays are converted to visible light by a $300 \mu \mathrm{m}$ thick LuAG:Ce scintillator. This configuration resulted in a scattering vector range between 0.872 and $5.23 \mu \mathrm{m}^{-1}$. (a)

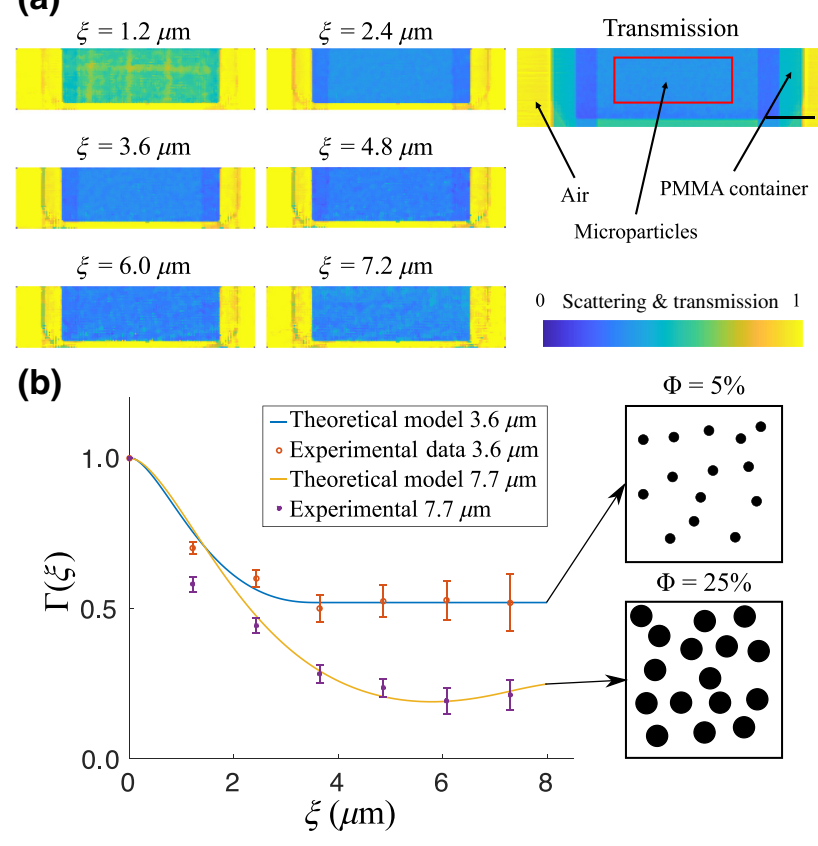

FIG. 3. Validation of the real space autocorrelation function retrieval. (a) Retrieved scattering and transmission images of the $7.7 \mu \mathrm{m}$ particles (scale bar $2.5 \mathrm{~mm}$ ). (b) Retrieved real space correlation functions of the two investigated cases within the marked area in the transmission image. The error bars correspond to the standard deviation of the retrieved scattering signals.

For validation, we image $\mathrm{SiO}_{2}$ microparticles [26] suspended in glycerine. The glycerin is used in order to keep the particles suspended and avoid gravitational sedimentation. Two scenarios are investigated: one with a low concentration (volume fraction $\Phi=5 \%$ ) of $d=3.6 \mu \mathrm{m}$ diameter particles, and one with a high concentration $(\Phi=25 \%)$ of $d=7.7 \mu \mathrm{m}$ diameter particles. The particle suspensions are placed in a PMMA container with a

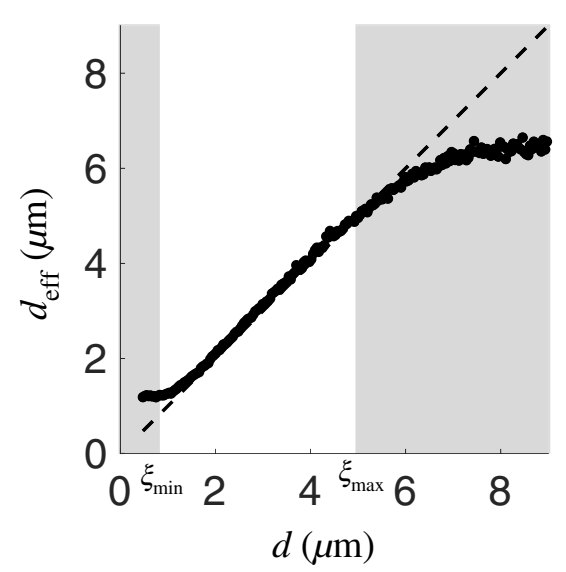

FIG. 4. Sensitivity curve for particle size estimation. The gray areas correspond to particles smaller or larger than $\xi_{\min }$ and $\xi_{\max }$, respectively. 


\section{(a)}

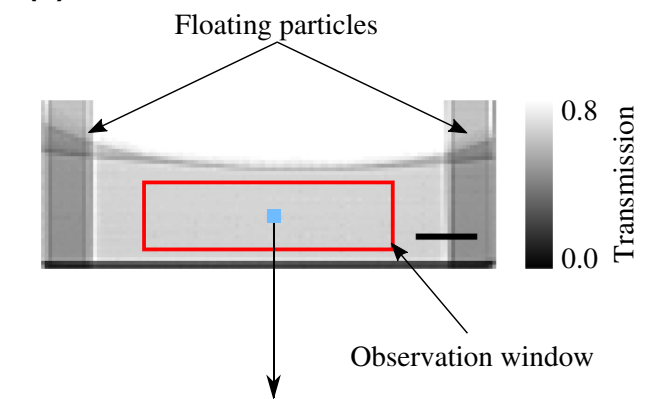

(c)

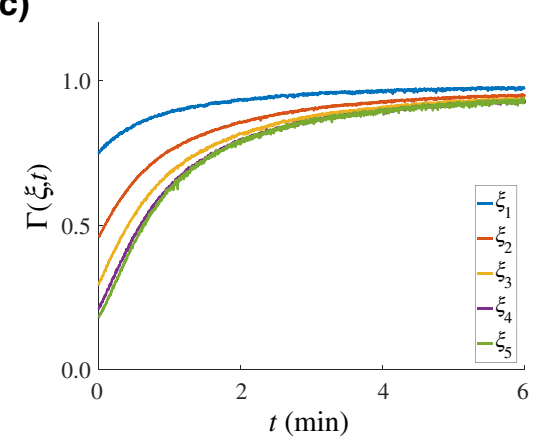

(b)

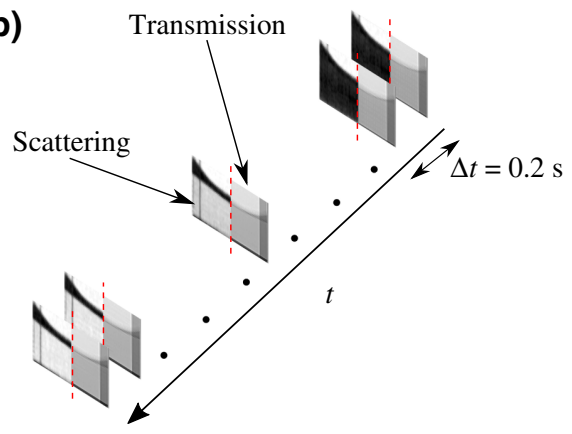

(d)

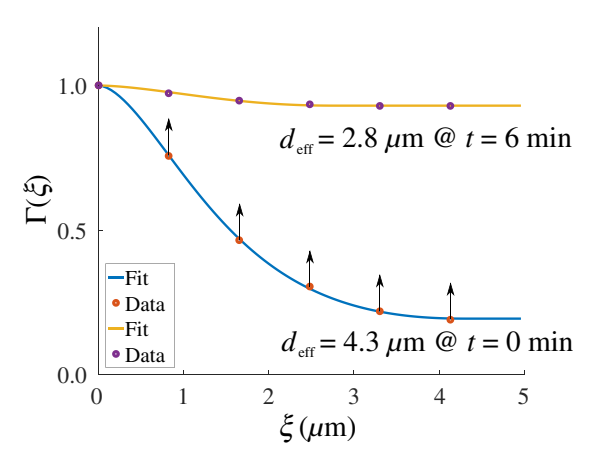

FIG. 5. Dynamic imaging of the polydisperse particle solution. (a) Transmission image of the particle solution at the end of the measurement. The red area denotes the observation window (scale bar $1.5 \mathrm{~mm}$ ). (b) Ensemble of transmission and scattering frames recorded at $0.2 \mathrm{sec}$ intervals. (c) Retrieved scattering intensities at the light blue are marked in transmission image (a). At each time point the scattering intensities are used to fit the effective particle diameter. (d) Fitted curves at time points $t=0$ min and $t=6$ min. The arrows indicate time evolution.

thickness of $4 \mathrm{~mm}$. In Fig. 2 we show the raw images for the case of particles with a diameter of $7.7 \mu \mathrm{m}$. In Fig 3(a) we show the retrieved transmission and scattering images for the $7.7 \mu \mathrm{m}$ suspension. The autocorrelation map at $\xi=1.2 \mu \mathrm{m}$ shows a periodic pattern that can be attributed to stitching errors in the fabrication of the zone plates. As expected, the suspension is strongly scattering while the PMMA container is only visible in the transmission images. In Fig. 3(b) we compare the retrieved real space correlation functions to theoretical ones that are calculated in SasView [27], assuming a hard sphere model for the structure factor. In particular, we simulated smallangle scattering patterns in a $q$ range between $10^{-2}$ and $10^{2} \mu \mathrm{m}^{-1}$ of monodisperse spheres with diameters of 3.6 and $7.7 \mu \mathrm{m}$. The scattering patterns are transformed into real space correlation functions by the Hankel transform. Good agreement is observed for both scenarios. For the low concentration case, the signal saturates when $\xi \geq d$; for the higher concentration, an oscillation can be observed due to interparticle correlations. Both observations are in good agreement with previous work $[18,28]$. Furthermore, at the lower correlation range a higher deviation from the theoretical values is observed. This could be an effect of the parasitic zeroth order of the zone plates. The etching depth of each zone is highly dependent on the width of the zone [29], meaning that different etch depths will occur for different zones. The nonuniform etching depth could be mitigated by utilizing the recently developed method of metal assisted chemical etching [30].

\section{DYNAMIC IMAGING}

To highlight the time-resolved capabilities of the method, we image the flotation process in an unstable polydisperse particle solution. As particles, we utilize 110P8 hollow microspheres with diameters ranging from 1 to $25 \mu \mathrm{m}$ of fused Borosilicate glass [31]. The solution is prepared by mixing $0.4 \mathrm{~g}$ of hollow spheres with $3 \mathrm{ml}$ of deionised water in a $5 \mathrm{ml}$ test vial. The vial is vortexed for $5 \mathrm{~min}$ prior to imaging. A volume of $1 \mathrm{ml}$ of the mixed solution is pipetted into a PMMA container. The imaging started approximately $30 \mathrm{sec}$ after the pipetting. The solution is imaged for a total of 1716 frames acquired with a frequency of $5 \mathrm{~Hz}$, resulting in a total measurement time of about $6 \mathrm{~min}$. The experimental parameters are summarized in Table I. In Fig. 5(b) an ensemble of collage images highlights the evolution of the process, where the right half corresponds to transmission and the left half to scattering at correlation length $\xi_{\max }$.

Assuming that particles with different sizes contribute incoherently to the scattering signal, we can estimate an effective particle size by fitting the monodispersed model 
(a)

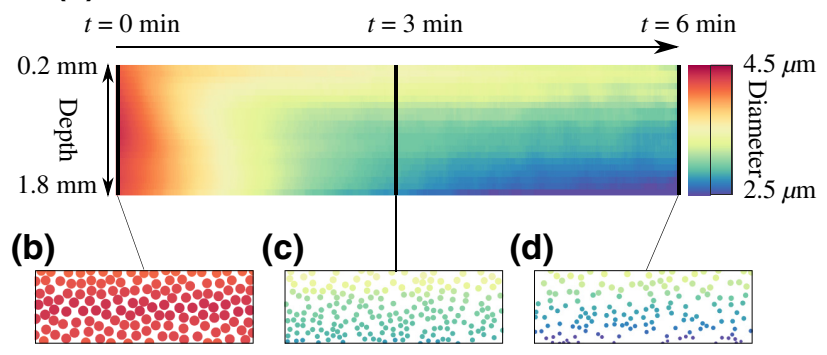

FIG. 6. Evolution of the depth-dependent effective particle diameter. (a) Effective particle diameter averaged along the observation window highlighted in Fig. 5(a). (b),(c),(d) Representative illustrations of the effective particle diameter at different time points.

[32] on our data. To corroborate the claim that the particle size can be estimated from the retrieved autocorrelation function containing a few sample points, we perform a set of wave optics simulations. We compare the retrieved particle size for a large number of particle distributions with diameters ranging from 0.45 to $9 \mu \mathrm{m}$ with a step size of $40 \mathrm{~nm}$. In Fig. 4 the resulting sensitivity curve for our experimental setup is shown. We can see that, for particles within the sensitivity range, a reliable estimation is expected. Details regarding the simulation are given in the Supplemental Material [25].

In order to fulfill the condition of uncorrelated particles of our model, we analyse the central area of the container, as annotated in Fig. 5(a), where no close packing between the particles takes place. In Fig. 5(c) the scattering signal at the unit cell corresponding to the light blue location is plotted over time for the five correlation lengths. At each time point an effective diameter is fitted, as shown in Fig. 5(d).

In Fig. 6(a) the average depth-dependent effective diameter over the marked area is shown for the duration of the experiment. Initially, a homogeneous effective diameter is observed; a representation of the effective diameter is shown in Fig. 6(b). The color encodes the effective particle diameter according to the colorbar in Fig. 6(a), the diameter is proportional to the effective diameter, and the density is proportional to the estimated normalized scattering cross section that is proportional to the volume fraction $\Phi$. After the first few minutes a gradient in the effective diameter starts to develop, as shown in Fig. 6(c), which gets increasingly steeper until the end of the observation period [Fig. 6(d)]. A movie with the evolution of the effective diameter is provided in the Supplemental Material [25]. The basis for explaining this gradient is the dependence of the flotation speed with the particle size that is a derivative of Stokes' law. In general, the flotation velocity $v$ is proportional to the square of the particle radius $v \propto r^{2}$. The observed behavior is supported by published numerical and mathematical studies describing such processes [33-36].

\section{CONCLUSION}

We have proposed an x-ray imaging method that allows simultaneous reciprocal and real space imaging in a single shot. The method is applied for imaging unstable polydisperse microparticle solutions. Because of the ability to achieve simultaneously high temporal resolution, spatial resolution, and large imaging area, we are able to observe the evolution of the local average particle size. Limitations of current characterization methods such as small angle x-ray scattering, dynamic light scattering and ultramicroscopy have not allowed direct measurements at this scale before.

The smallest structure that can be quantified by our method is defined by the minimum correlation length $\xi_{\min }$, which is equal to the outermost zone width of the zone plates $\Delta r$. With state-of-the-art fabrication methods, outer most zone widths between 7 [37] and $40 \mathrm{~nm}$ [38] for 9 and $20 \mathrm{keV}$, respectively, have been reported, opening up the potential for nanoscale sensitivity over a wide range of $\mathrm{x}$-ray energies.

The main limitation of the proposed method is the number of sampling points within each macropixel. This inevitably limits the details in the recorded scattering patterns. Therefore, we do not make the claim that this method is a replacement of the conventional (scanning) small angle $\mathrm{x}$-ray scattering methodology. But, it shall rather be viewed as a tool that extends the capabilities of full-field imaging beamlines, providing quantitative subpixel information for macroscopic samples in a single shot. Finally, we would like to point out that the approach is not beamline dependent and could easily be translated to other imaging beamlines or even highly coherent laboratory sources such as liquid metal jet sources.

\section{ACKNOWLEDGMENTS}

The imaging experiments were performed at the TOMCAT beamline of the Swiss Light Source, PSI Villigen, Switzerland. The fabrication of the optical elements was performed at the laboratory of micro and nanofabrication $(\mathrm{LMN})$, PSI Villigen, Switzerland. Part of this work is funded by the Eurostars Grant E!1106 INFORMAT, SNF Sinergia Grant CRSII5_18356 "Clinical GI-BCT," and the lottery fund SwissLOS of the Canton of Aargau.

\section{APPENDIX: IMAGE FORMATION}

The purpose of this derivation is to demonstrate that each Fresnel zone plate acts as a local Fourier transform of the complex transmission of the sample. For the derivation, we assume fully coherent and monochromatic plane wave illumination. For the theoretical description, we define 
the following local two-dimensional coordinate systems corresponding to each unit cell $(m, n)$ of our optics; for simplicity, the $m, n$ indexing is omitted. The experimental setup together with all definitions of local coordinates are shown in Fig. 1(a). For a concise description, we utilize vector notation for each plane, assuming that the center of each unit cell corresponds to the local origin. The defined coordinate systems are the spatial coordinate at the sample plane and optical element plane $\mathbf{r}$; and the spatial coordinate at the detector plane $\mathbf{s}$. We assume that our local zone plates are $\pi$ phase shifting in order to eliminate the zeroth diffraction order. The complex transmission function $Z(\mathbf{r})$ of a zone plate can be decomposed into a Fourier series as [39]

$$
\begin{aligned}
Z(\mathbf{r}) & =\operatorname{sign}\left[\cos \left(\frac{2 \pi}{2 \lambda f}|\mathbf{r}|^{2}\right)\right] \\
& =\sum_{n= \pm 1, \pm 3, \ldots}^{ \pm \infty} \frac{2}{|n| \pi}(-1)^{(|n|-1) / 2} e^{i 2 \pi|\mathbf{r}|^{2} n / r_{0}^{2}}
\end{aligned}
$$

where $f$ is the focal distance of the Fresnel zone plate, $\lambda$ is the design wavelength, and $r_{0}^{2}=2 \lambda f$. This expansion is achieved by exploiting the circular symmetry of the transmission function and performing the Fourier decomposition as if the function is a one-dimensional signal of period $r_{0}^{2}$, with $r^{2}=|\mathbf{r}|^{2}$ being the independent variable. Assuming fully coherent monochromatic plane wave illumination of wavelength $\lambda$, the propagated image of the complex transmission function $t(\mathbf{r})$ of the sample (placed immediately before the optical element) at distance $z$ from the optical element can be calculated by utilizing the Fresnel propagator:

$$
\begin{aligned}
T(\mathbf{s}, z)= & \int d \mathbf{r} t(\mathbf{r}) Z(\mathbf{r}) e^{i \pi|\mathbf{r}-\mathbf{s}|^{2} / \lambda z} \\
= & \int d \mathbf{r} t(\mathbf{r}) \sum_{n= \pm 1, \pm 3, \ldots}^{ \pm \infty} \frac{2}{|n| \pi}(-1)^{(|n|-1) / 2} e^{i 2 \pi|\mathbf{r}|^{2} n / r_{0}^{2}} \\
& \times e^{i \pi|\mathbf{r}-\mathbf{s}|^{2} / \lambda z}
\end{aligned}
$$

By expanding the term $|\mathbf{r}-\mathbf{s}|^{2}$ and setting $z=f$ since we are interested in the image formed at the focal plane of the zone plates, Eq. (A2) becomes

$$
\begin{aligned}
T(\mathbf{s}, f)= & e^{i \pi|\mathbf{s}|^{2} / \lambda z} \\
& \times \int d \mathbf{r} e^{i 2 \pi \mathbf{r} \mathbf{s} / \lambda f} \sum_{n= \pm 1, \pm 3, \ldots}^{ \pm \infty} t(\mathbf{r}) \frac{2}{|n| \pi}(-1)^{(|n|-1) / 2} \\
& \times e^{i \pi|\mathbf{r}|^{2}(n+1) / \lambda f}
\end{aligned}
$$

We define the family of functions

$$
\begin{aligned}
t_{n}(\mathbf{r}) & =t(\mathbf{r}) \frac{2}{|n| \pi}(-1)^{(|n|-1) / 2} e^{i \pi|\mathbf{r}|^{2}(n+1) / \lambda f}, \\
n & \in\{ \pm 1, \pm 3, \ldots\} .
\end{aligned}
$$

Hence, Eq. (A3) can be rewritten as

$$
T(\mathbf{s}, f)=e^{i \pi|\mathbf{s}|^{2} / \lambda z} \int d \mathbf{r} e^{i 2 \pi \mathbf{r} / \lambda f} \sum_{n= \pm 1, \pm 3, \ldots}^{ \pm \infty} t_{n}(\mathbf{r}) .
$$

The integral part of Eq. (A5) represents the Fourier transform of the infinite sum of the functions $t_{n}(\mathbf{r})$. Given the linearity of the Fourier transform and under the assumption that the $t_{n}(\mathbf{r})$ are square integrable (existence of the Fourier transform), the order of summation and integration can be exchanged in Eq. (A5). The assumption of square integrability,

$$
\int d \mathbf{r}\left|t_{n}(\mathbf{r})\right|^{2}=\int d \mathbf{r}|t(\mathbf{r})|^{2} \frac{4}{|n|^{2} \pi^{2}}<\infty,
$$

holds for realistic samples with a finite support. Equation (A5) can now be rewritten as

$$
T(\mathbf{s}, f)=e^{i \pi|\mathbf{s}|^{2} / \lambda z} \sum_{n= \pm 1, \pm 3, \ldots}^{ \pm \infty} \hat{t}_{n}\left(\mathbf{s} \frac{k}{f}\right),
$$

where $\hat{t}_{n}(\mathbf{s} k / f)$ represents the Fourier transform of $t_{n}(\mathbf{r})$ and $k=2 \pi / \lambda$. Each of these terms corresponds to a diffraction order of the zone plate. From Eqs. (A4) and (A5), it follows that $\hat{t}_{-1}(\mathbf{s} k / f)$ is the Fourier transform of the sample $t(\mathbf{r})$. For the higher orders $|n| \geq 3$, we assume that

$$
\left|\hat{t}_{-1}\left(\mathbf{s} \frac{k}{f}\right)\right|^{2} \gg\left|\hat{t}_{n}\left(\mathbf{s} \frac{k}{f}\right)\right|^{2}
$$

since the intensity drops with $1 /|n|^{2}$. Additionally, under the assumption that our sample is locally varying slower than $2|\mathbf{r}|^{2}$, the +1 term can also be neglected since it converges to 0 . Finally, this leads to the recorded intensity by the $\mathrm{x}$-ray detector being

$$
I(\mathbf{s}) \propto\left|\hat{t}\left(\mathbf{s} \frac{k}{f}\right)\right|^{2} .
$$

Therefore, we have demonstrated that reciprocal information can be obtained on a local level, while maintaining spatial resolution on a large scale. Up to this point in our derivation fully coherent illumination and ideal response from the x-ray detector have been assumed. Nonfully coherent illumination can be incorporated through the Van 
Cittert-Zernike theorem [40], stating that the recorded intensity should be convolved with the projected source distribution $S(\mathbf{s})$. To incorporate the response of the imaging system, a second convolution needs to be performed with the point spread function $\operatorname{PSF}(\mathbf{s})$ of the detector. Therefore, the final recorded signal is

$$
I(\mathbf{s}) \propto\left|\hat{t}\left(\mathbf{s} \frac{k}{f}\right)\right|^{2} * \operatorname{PSF}(\mathbf{s}) * S(\mathbf{s}) .
$$

By having sufficient knowledge of the imaging system and the x-ray source, deconvolution algorithms could be applied in order to retrieve the scattering distribution. However, taking into account the limited number of sample points within each unit cell, a more beneficial approach would be to study the Fourier transform of the scattering intensity, which is known as the real space correlation function

$$
G(\xi)=\left|\frac{f}{k}\right|^{2} t\left(\xi \frac{f}{k}\right) * t^{\star}\left(\xi \frac{f}{k}\right),
$$

where $\boldsymbol{\xi}$ is the reciprocal variable of $\mathbf{s}$ and $t^{\star}$ the conjugate of $t$. By performing a reference measurement with only the optical element in the beam, the effect of the finite source size and PSF of the detector can be compensated in the real space correlation function.

So far, we demonstrated that reciprocal space information can be obtained on a local scale while maintaining spatial resolution on a large scale; here we derive the relation between the two for an arbitrary optical element design. Let $R_{N}$ be the radius of each zone plate with focal length $f$, and let $\Delta x$ be the sampling rate of our imaging detector. The maximum value of the scattering vector $\mathbf{q}$ depends on the size of the unit cell and is given by

$$
q_{\max }=|\mathbf{q}|_{\max }=4 \frac{\pi}{\lambda} \frac{|\mathbf{s}|_{\max }}{f}=4 \frac{\pi}{\lambda} \frac{R_{N}}{f} .
$$

By knowing the sampling rate $\Delta x$ we can obtain the maximum autocorrelation length

$$
\xi_{\max }=\lambda f /(2 \Delta x),
$$

and the sampling rate of the autocorrelation function, which is equal to the minimum autocorrelation length $\xi_{\text {min }}$ of the system

$$
\Delta \xi=\xi_{\min }=\lambda f /\left(2 R_{N}\right),
$$

which is also equal to the width $\Delta r$ of the outermost zone of the zone plate. From the above equations, we can formulate an uncertainty principle between the spatial resolution $\Delta X$ of the obtained image and the resolution $\Delta \xi$ with which the real space correlation function is sampled.
The spatial resolution $\Delta X$ has a minimum equal to the diameter of the zone plate

$$
\Delta X \geq 2 R_{N} .
$$

From Eqs. (A14) and (A15) we conclude that

$$
\Delta X \Delta \xi \geq \lambda f,
$$

meaning that, for a given focal length and wavelength, higher sampling of the real space correlation function can only be achieved by reducing the real space resolution.

[1] T. A. Schaedler, A. J. Jacobsen, A. Torrents, A. E. Sorensen, J. Lian, J. R. Greer, L. Valdevit, and W. B. Carter, Ultralight metallic microlattices, Science 334, 962 (2011).

[2] Sanghyuk Wooh, Hannah Huesmann, Muhammad Nawaz Tahir, Maxime Paven, Kristina Wichmann, Doris Vollmer, Wolfgang Tremel, Periklis Papadopoulos, and Hans-Jürgen Butt, Synthesis of mesoporous supraparticles on superamphiphobic surfaces, Adv. Mater. 27, 7338 (2015).

[3] Changbao Zhu, Robert E. Usiskin, Yan Yu, and Joachim Maier, The nanoscale circuitry of battery electrodes, Science 358, eaao2808 (2017).

[4] Kim Nygård, Johan Buitenhuis, Matias Kagias, Konstantins Jefimovs, Federico Zontone, and Yuriy Chushkin, Anisotropic de Gennes Narrowing in Confined Fluids, Phys. Rev. Lett. 116, 167801 (2016).

[5] D. K. Satapathy, O. Bunk, K. Jefimovs, K. Nygård, H. Guo, A. Diaz, E. Perret, F. Pfeiffer, C. David, G. H. Wegdam, and J. F. van der Veen, Colloidal Monolayer Trapped near a Charged Wall: A Synchrotron $X$-Ray Diffraction Study, Phys. Rev. Lett. 101, 136103 (2008).

[6] Rei Kurita, David B. Ruffner, and Eric R. Weeks, Measuring the size of individual particles from three-dimensional imaging experiments, Nat. Commun. 3, 1127 (2012).

[7] Yoshihiro Nagasawa, Zenji Kato, and Satoshi Tanaka, Particle sedimentation monitoring in high-concentration slurries, AIP Adv. 6, 115206 (2016).

[8] Atsushi Momose, Shinya Kawamoto, Ichiro Koyama, Yoshitaka Hamaishi, Kengo Takai, and Yoshio Suzuki, Demonstration of $X$-Ray talbot interferometry, Japanese J. Appl. Phys. 42, L866 (2003).

[9] Alessandro Olivo and Robert Speller, A coded-aperture technique allowing $x$-ray phase contrast imaging with conventional sources, Appl. Phys. Lett. 91, 074106 (2007).

[10] Kaye S. Morgan, David M. Paganin, and Karen K. W. Siu, $X$-ray phase imaging with a paper analyzer, Appl. Phys. Lett. 100, 124102 (2012).

[11] F. Pfeiffer, M. Bech, O. Bunk, P. Kraft, E. F. Eikenberry, Ch. Brönnimann, C. Grünzweig, and C. David, Hard- $X$ ray dark-field imaging using a grating interferometer, Nat. Mater. 7, 134 (2008).

[12] Marco Endrizzi, Paul C. Diemoz, Thomas P. Millard, J. Louise Jones, Robert D. Speller, Ian K. Robinson, and Alessandro Olivo, Hard $X$-ray dark-field imaging with incoherent sample illumination, Appl. Phys. Lett. 104, 024106 (2014). 
[13] Hongchang Wang, Yogesh Kashyap, and Kawal Sawhney, Hard- $X$-Ray Directional Dark-Field Imaging Using the Speckle Scanning Technique, Phys. Rev. Lett. 114, 103901 (2015).

[14] Matias Kagias, Zhentian Wang, Mie Elholm Birkbak, Erik Lauridsen, Matteo Abis, Goran Lovric, Konstantins Jefimovs, and Marco Stampanoni, Diffractive small angle $X$ ray scattering imaging for anisotropic structures, Nat. Commun. 10, 5130 (2019).

[15] Matias Kagias, Zhentian Wang, Pablo Villanueva-Perez, Konstantins Jefimovs, and Marco Stampanoni, 2DOmnidirectional Hard- $X$-Ray Scattering Sensitivity in a Single Shot, Phys. Rev. Lett. 116, 093902 (2016).

[16] Susanna K. Lynch, Vinay Pai, Julie Auxier, Ashley F. Stein, Eric E. Bennett, Camille K. Kemble, Xianghui Xiao, WahKeat Lee, Nicole Y. Morgan, and Han Harold Wen, Interpretation of dark-field contrast and particle-size selectivity in grating interferometers, Appl. Opt. 50, 4310 (2011).

[17] P. Modregger, F. Scattarella, B. R. Pinzer, C. David, R. Bellotti, and M. Stampanoni, Imaging the Ultrasmall-Angle $X$-Ray Scattering Distribution with Grating Interferometry, Phys. Rev. Lett. 108, 048101 (2012).

[18] Ralph P. Harti, Markus Strobl, Benedikt Betz, Konstantins Jefimovs, Matias Kagias, and Christian Grünzweig, Subpixel correlation length neutron imaging: Spatially resolved scattering information of microstructures on a macroscopic scale, Sci. Rep. 7, 44588 (2017).

[19] Matias Kagias, Zhentian Wang, Konstantins Jefimovs, and Marco Stampanoni, Dual phase grating interferometer for tunable dark-field sensitivity, Appl. Phys. Lett. 110, 014105 (2017).

[20] Jacopo Valsecchi, Markus Strobl, Ralph Patrick Harti, Chiara Carminati, Pavel Trtik, Anders Kaestner, Christian Grünzweig, Zhentian Wang, Konstantins Jefimovs, and Matias Kagias, Characterization of oriented microstructures through anisotropic small-angle scattering by 2D neutron dark-field imaging, Commun. Phys. 3, 42 (2020).

[21] G. Harburn and R. P. Williams, On images and fraunhofer diffraction patterns obtained from fresnel zone plates, Optica Acta: Int. J. Opt. 22, 37 (1975).

[22] Timofei Krouglov, Ignatz M. de Schepper, Wim G. Bouwman, and M. Theo Rekveldt, Real-space interpretation of spin-echo small-angle neutron scattering, J. Appl. Crystallogr. 36, 117 (2003).

[23] Timofei Krouglov, Wim G. Bouwman, Jeroen Plomp, M. Theo Rekveldt, Gert Jan Vroege, Andrei V. Petukhov, and Dominique M. E. Thies-Weesie, Structural transitions of hard-sphere colloids studied by spin-echo small-angle neutron scattering, J. Appl. Crystallogr. 36, 1417 (2003).

[24] W. Yashiro, Y. Terui, K. Kawabata, and A. Momose, On the origin of visibility contrast in $x$-ray talbot interferometry, Opt. Express 18, 16890 (2010).

[25] See Supplemental Material at http://ink.aps.org/supple mental/10.1103/PhysRevApplied.15.044038 for fabrication and characterization details of optical elements, wave optics simulations for effective diameter fitting, a movie with the retrieved effective diameter sped up 33 times, which includes Ref. [41].

[26] Cospheric LLC, USA, https://www.cospheric.com/SiO2MS _monodisperse_silica_spheres_beads_nm_microns.htm.
[27] This work benefited from the use of the SasView application, originally developed under NSF Grant DMR0520547. SasView contains code developed with funding from the European Union's Horizon 2020 research and innovation programme under the SINE2020 project, Grant No 654000. See https://www.sasview.org/.

[28] Spyridon Gkoumas, Pablo Villanueva-Perez, Zhentian Wang, Lucia Romano, Matteo Abis, and Marco Stampanoni, A generalized quantitative interpretation of darkfield contrast for highly concentrated microsphere suspensions, Sci. Rep. 6, 35259 (2016).

[29] Junghoon Yeom, Yan Wu, John C. Selby, and Mark A. Shannon, Maximum achievable aspect ratio in deep reactive ion etching of silicon due to aspect ratio dependent transport and the microloading effect, J. Vac. Sci. Technol. B: Microelectron. Nanometer Struct. 23, 2319 (2005).

[30] Lucia Romano, Matias Kagias, Joan Vila-Comamala, Konstantins Jefimovs, Li-Ting Tseng, Vitaliy A. Guzenko, and Marco Stampanoni, Metal assisted chemical etching of silicon in gas phase: a nanofabrication platform for $X$-ray optics, Nanoscale Horizons (2020), 10.1039/C9NH00709A.

[31] PQ Corporation, https://www.ulprospector.com/en/na/Coa tings/Detail/25921/572868/Sphericel-110P8.

[32] M. Strobl, General solution for quantitative dark-field contrast imaging with grating interferometers, Sci. Rep. 4, 7243 (2015).

[33] Nhan-Quyen Nguyen and Anthonyj C. Ladd, Sedimentation of hard-sphere suspensions at low reynolds number, J. Fluid Mech. 525, 73 (2005).

[34] Stefano Buzzaccaro, Antonio Tripodi, Roberto Rusconi, Daniele Vigolo, and Roberto Piazza, Kinetics of sedimentation in colloidal suspensions, J. Phys.: Condens. Matter 20, 494219 (2008).

[35] David K. Basson, Stefan Berres, and Raimund Bürger, On models of polydisperse sedimentation with particle-sizespecific hindered-settling factors, Appl. Math. Model. 33, 1815 (2009).

[36] Evangelia Antonopoulou, Connor F. Rohmann-Shaw, Thomas C. Sykes, Olivier J. Cayre, Timothy N. Hunter, and Peter K. Jimack, Numerical and experimental analysis of the sedimentation of spherical colloidal suspensions under centrifugal force, Phys. Fluids 30, 030702 (2018).

[37] Istvan Mohacsi, Ismo Vartiainen, Benedikt Rösner, Manuel Guizar-Sicairos, Vitaliy A. Guzenko, Ian McNulty, Robert Winarski, Martin V. Holt, and Christian David, Interlaced zone plate optics for hard $X$-ray imaging in the $10 ? \mathrm{~nm}$ range, Sci. Rep. 7, 43624 (2017).

[38] Takahisa Koyama, Hidekazu Takano, Shigeki Konishi, Takuya Tsuji, Hisataka Takenaka, Satoshi Ichimaru, Tadayuki Ohchi, and Yasushi Kagoshima, Circular multilayer zone plate for high-energy $x$-ray nano-imaging, Rev. Sci. Instrum. 83, 013705 (2012).

[39] Wood Robert, Physical Optics (The Macmillan Company, New York, 1934), p. 907.

[40] F. Zernike, The concept of degree of coherence and its application to optical problems, Physica 5, 785 (1938).

[41] T. Hildebrand, and P. Ruegsegger, A new method for the model-independent assessment of thickness in threedimensional images, J. Microsc. 185, 67 (1997). 